Port abi I i ty i mprovement of LABCOM dat a acqui si t $i$ on system for the next-gener at $i$ on fusi on experi ment $s$

\begin{tabular}{|c|c|}
\hline $\begin{array}{l}\text { jour nal or } \\
\text { publ i cat } i \text { on title }\end{array}$ & Fusi on Engi neer ing and Desi gn \\
\hline vol une & Vol . 82 \\
\hline number & I ssues $5-14$ \\
\hline page $r$ ange & pp. 1203- 1209 \\
\hline year & 2007- 10-01 \\
\hline URL & ht t p: //hdl . handl e. net /10655/333 \\
\hline
\end{tabular}




\title{
Portability Improvement of LABCOM Data Acquisition System for the Next-Generation Fusion Experiments
}

\author{
NAKANISHI, Hideya, OHSUNA, Masaki, KOJIMA, Mamoru, \\ IMAZU, Setsuo $^{\text {b }}$, NONOMURA, Miki, NAGAYAMA, Yoshio, \\ and KAWAHATA, Kazuo
}

National Institute for Fusion Science, 322-6 Oroshi-cho, Toki 509-5292, Japan

${ }^{\mathrm{b}}$ Pretech Corp., 1-19-13 Kanayama-cho, Atsuta-ku, Nagoya 456-0002, Japan

\begin{abstract}
The LABCOM data acquisition system (DAQ) has set a new world record of acquired data amount of $90 \mathrm{~GB} /$ shot in 2005-2006 campaign. Its CompactPCI-based new DAQ enables about $80 \mathrm{MB} / \mathrm{s}$ real-time acquisition for each plasma measurement. The basic performance of a DAQ unit has already good prospects for the next-generation fusion experiments, however, some surrounding utilities are not necessarily developed to construct tentimes larger DAQ system yet. The increasing number of parallel DAQ units has made the operational and maintenance burden very heavy. So, "more distributed acquisition and centralised operations" would become expected. Here, DAQ front-end consisting of some digitizer chassis, a timing module, and an acquisition computer has been entirely re-designed to realize a lower-cost and maintenance-free "DAQ Box" for fusion plasma measurements. OS and softwares have been also remodelled to use free drivers on Linux. Network-bootable diskless computers are also desirable for reducing the possibility of hardware troubles, and it simultaneously realizes to omit first-tier data storage and reduce the migration operational cost. As a result, total hardware cost could be reduced almost one fifth than the previous. This could be the way to realize ten-times larger fusion experiment in the near future.
\end{abstract}

Key words: LABCOM/X, LHD, DAQ Box, network-bootable diskless PC, digital timing system, zip archive, JPEG-LS, DataGrid, ITBL

Email address: nakanishi.hideya@lhd.nifs.ac.jp (NAKANISHI, Hideya). 


\section{Introduction}

The LABCOM data acquisition system (DAQ) has been operated for the LHD plasma diagnostics for nine years. It has set a new world record of acquired data amount of $90 \mathrm{~GB} /$ shot in 2005-2006 campaign (Fig. 1). Its ultra-wideband DAQ based on the CompactPCI digitizers has enabled about $80 \mathrm{MB} / \mathrm{s}$ real-time data acquisition for each plasma measurement [1]. Actually proved $80 \mathrm{MB} / \mathrm{s}$ bandwidth could easily satisfy the requirement for the advanced, large-scale fusion experiments, such as LHD and ITER. The basic performance of a DAQ unit has enough capability, however, the subsidiary utilities are not fully developed yet [2].

LHD diagnostic information system, including DAQ, is always expected to take the head of research and development in fusion experiments of the world, and even in such the ITER-BA remote experiment center and All-Japan ST research program [3]. Even though LHD renewed the world record of acquired data amount in 2006, however, the tendency of substantial data increase is worldwide common in these several years. As this problem becomes quite remarkable in large scale experiments, innovation of data processing technology is urgently needed to be fit for their practical purposes.

LHD already had over sixty DAQs in the 9th campaign. Its increasing number of parallel DAQ units has made the operational and maintaining burden quite heavy. In addition, the data migration procedures between multi-tier distributed storages also need much closer attention. Therefore, "more distributed acquisition and more centralised operations" would be indispensable to cope with both high-efficiency I/O throughputs and much enlarged data volume.

In this study, DAQ front-end that consists of some digitizer chassis and an acquisition computer has been entirely re-designed to realize lower costs and lesser maintenance/operation frequency for fusion plasma measurement.

\section{Conceptual Remodelling of DAQ Framework}

Modern fusion experiments often have a few tens of plasma measurement racks. LHD, the leading new and powerful experiment, has already seventy in 2006. In order to make sure of the scalability of such a increasing number of DAQs up to a thousand, for the next-generation experiment, we have to consider the following issues;

(1) Cost-cutting and downsizing of DAQ elements

(2) Far scalability and more concentrated operation

(3) Multi-site logical extension ... DataGrid 
(4) Remote experiment based on "Long Fat pipe Network (LFN)" [4-6].

The cost reduction should be considered on both of hardware and software. So, we first discuss about dismissing the commercial softwares; OS, DBMS, and IDE (Integrated Development Environment).

$O S$

We have developed our DAQ system wholly on Windows (Win32) before. It was simply because digitizer drivers and APIs of CAMAC, Yokogawa WE7000, and NI PXI were provided only for Windows. At present July 2006, this situation still remains partly, for instance, in NI-IMAQ image grabbing driver and API. However, Linux drivers and APIs become available for most of the other digitizers.

Another important requirement for OS is to perfectly support the network booting and diskless clients. Since the LABCOM DAQ system has the distributed architecture, the DAQ host would be installed in proportion to the increase of the number of LHD plasma measurement. Its number has already exceeded sixty in the end of 2005-2006 campaign, and will be almost seventy in the next one. Human works to setup OS and DAQ applications in such large number of PCs and to keep their conditions have become gradually heavier for the definite number of operators.

Once MS Windows intended to provide network booting and diskless clients, however, at present we can seldom find any Windows XP or 2003 information about it. As a result, diskless Windows machine is very difficult now. On the other hand, Linux already has a well managed environment for the "thin client" setup. diskless configuration is even very easy, if we have PXE capable machine with appropriate NIC and BIOS.

With the following reasons, we decided to switch our standard OS from MS Windows to Linux;

- By managing a single boot image, numbers of hosts can be booted on network.

- It supports the diskless environment very well.

- Free of charge, Even though a thousand of hosts installed it.

\section{$O D B M S$}

Inside the DAQ processing program of LABCOM system, measured signals are handled as the on-memory instance of the $\mathrm{C}++$ data object. It is very straightforward to use the object-oriented database management system (ODBMS) to make the volatile instance persistent, which can transparently store them into hard-drive as it is on memory. Until now, LABCOM system has been using two ODBMS successively; $\mathrm{O} 2$ and ObjectStore. 
Naturally, their initial price and volume license fee are so expensive that we hardly apply them to a huge system of one thousand cpu. It never fit our new approach of open and free software. Here, we will modify the DAQ programs not to use ODBMS. Instead, objects would be transformed into files in usual file-system where the I/O performance is very sure.

We still keep an interest in the approach to make a specific software optimized for BLOB housing for the future study.

$I D E$

Until this study, our development environment completely depended on commercial products; MS Visual Studio for $\mathrm{C}++$, Visual J++ for Java, and Visual SourceSafe for code rivisioning. It was because out DAQ system were all worked on MS Windows (Win32).

To introduce free softwares instead,

- Sun Open Studio or Eclipse

- CVS or subversion

- MS Visual C++ Express Ed., Intel C++ compiler, GCC

are available. After some trials, we have decided to adopt Eclipse, subversion, and GCC/Intel C++ compiler as our standard IDE.

\section{Downsizing of DAQ Elements}

Having above sixty parallel DAQs, we have well experienced that the unit reliability directly affects the frequency of trouble operations. To suppress the rise in the operation load, the unit reliability and stability must be improved than the previous. We have to find hardware or technology with low reliability, and replace it with higher one.

As the parallel distributed processing architecture originally has a high expandability, it can flexibly respond to the request of increase and reinforcement from users. However, if its maintenance cost are raised in proportion to its increasing number, it practically has the expansion limitation due to the annual operation budget. Maintenance and initial cost reduction of the DAQ unit are indispensable to be free from this constraint.

By improving the parts of frequent disorder to be free from maintenance and reducing the unit cost used in great numbers, we could obtain a new DAQ system which sufficiently enables the next-generation larger-scale experiment. 
To satisfy those conditions mentioned above, network-bootable diskless computers are desired for reducing the possibility of hardware troubles against the bad noise environment. To wire it adjacently to the digitizer controller could get rid of the cost for electrically isolating uplinks.

In addition, it would involve that the first-tier data storage become extinct, and therefore, could reduce the migration cost and time. Modification details about the storage structure will be mentioned in Section 4.

\subsection{DAQ Box}

For the cost reduction requirement, Linux OS and its free drivers for digitizers, such as CAMAC and CompactPCI, have been adopted instead of former Windowsbased system. It is also a very reliable environment to operate Network-bootable diskless computers.

The only point we first worried was less abundance of provided or distributed drivers for digitizer controllers. However, we fortunately have found that our main CAMAC, WE7000, and PXI digitizers have free Linux drivers, respectively. Especially, our inspection revealed that SCSI-USB2.0 conversion cables are the most promising in both transferring speed and the cost to replace the former SCSI optical extenders (Fig. 2).

For Kinetic 3929 and Jorway 73A CAMAC controllers, obtained data transferring speed were 1.18 and $1.6 \mathrm{MB} / \mathrm{s}$ with SCSI-USB conversion cables; Logitec LUB-SC2 and Adaptec USB2XCHANGE, respectively. Their improved factor are roughly double their original SCSI throughputs.

The cost comparison is much remarkable. As the SCSI optical extenders were nonstandard products, they were somewhat expensive with poor communication reliability (Table.1).

The remote power controller for the distributed digitizer crates and DAQ boxes is another stuff to lighten operator's burden. Daily power up and down of tens or hundreds of distributed crates would be quite hard work, and sometimes they accidentally become frozen during the experimental sequence.

In such cases, the network function of power recycling, once off then on, can help us not rushing to the equipment to reboot them. Easy power off and on capability also assist the power saving so much, as each CAMAC crate often consumes about $1 \mathrm{~kW}$ electricity. We have installed thirty sets of ISA PDU-5115S for every DAQ rack [8]. 


\subsection{Digital Timing Box}

The LHD diagnostic timing system had been developed as VMEbus modules at first, which are operated under VxWorks real-time OS [9]. We also developed CAMAC module version afterward, however, both of them need costly crate chassis and bus controllers indivisibly. VME ones additionally require expensive fee of software development license.

As this system has a simple tree structure on modulator-demodulator basis, most of the concrete problems concern a number of distributed demodulators. For replacing such the costly VME-based timing demodulator module, we have newly developed its FPGA version; FPGA-based timing demodulator box.

It adopts the embedded device "Suzaku-S" that combines the Spartan-3(E) FPGA and MicroBraze software processor [10]. As its SDK is distributed freely by Atmark Techno, Inc, FPGA logics have been newly rewritten by VHDL, and ONC RPC networking program also been ported to $\mu$ Clinux embedded environment.

As it consumes very small electricity $(3.3 \mathrm{~V} 350 \mathrm{~mA})$, there is no need having any cooling fan or large power supply in case. We can run it by a small AC adapter, and thus, this timing box will never need maintenance, free from any moving or rotating "fragile" parts.

Table 2 shows the outline of this new demodulator. The total cost of materials is less than $\$ 800$ for one demodulator box, which is almost one-eighth of the previous VME and CAMAC ones.

\section{Reconsideration of Data Storage}

\subsection{Reducing the Storage Tiers}

The diskless DAQ box also makes the first-tier data storage extinct. Acquired raw data are immediately compressed on memory, and written temporarily into files and directories on so-called RAMdisk filesystem by each channel and picture frame. As they are very volatile entities and the RAMdisk size are also limited, shot-byshot migration to the RAID storage is mandatory. After every migration have been finished normally, older shot directory and files will be deleted in order. Even in case of a sudden network accident, the acquisition operation could be continued for some more shots.

By giving up data accumulation on local hard-drive, we could reduce the batchprocessing migration procedures from the double action between three storage tiers 
to a single one (Fig. 3). It also could save our human cost and time for daily postexperimental works.

\subsection{Portable Archive Files}

A big fruit of our previous study was that we have found that the "JPEG-LS" is the most promising loss-less image compression algorithm in both the processing speed and the compression ratio [11]. It can deflate/inflate image data by frame to archive/retrieve one by one, even though "zlib" can only do the single-block compression.

It is very convenient for normal client PCs to retrieve image data by frame, because they do not have enough memory to process a full-length movie. Even in case of huge one-dimensional waveform data, dividing it into finer pieces would help us not dealing with huge objects on memory.

Our previous archive file format was non-standard private one. One DAQ produced one archive file for each shot, in which all the channel data are stored as a series of compressed blocks after the indexing header. There was no finer structure within a channel data block.

By thinking the faults of this archive format over, we have renewed its design as follows;

- Adopting the standard "zip" archive format

- Having shot/channel/frame sub-directory structure

- Meta-data are stored as a text file in every directory

- "zlib" compatible deflate()/inflate() for waveforms

- JPEG-LS compression for image frames

- Waveform/picture can be mixed in different channels.

The key feature we have chosen the zip format is that it has a file index to enable faster seek inside. Many other format, such as tar, does not have it.

As modern popular OS always have function or commands to do "zip/unzip", users can unzip the raw waveform data or JPEG-LS picture file from the archive, never worrying about the difference of working OS. It provides another good portability that JPEG-LS file (.jls) can be seen as it is by many picture browsers. 


\section{Summary and Future Work}

We have named this innovative project as "LABCOM/X" because it corresponds to its tenth revision toward the 10th LHD campaign. The results of this work can be confirmed concretely by comparing the total cost paid for one DAQ unit (Table. 3).

In CAMAC case, it is almost one fifth lower than the previous set with maintenancefree boxes. Other digitizers, WE7x and PXI, shares similar situation. This result shows very well that such the innovative approach can enable us to realize tentimes larger DAQ system in the near future, having one thousand DAQ PCs for plasma diagnostics.

Further, we try to give some discussion for the remaining subjects mentioned in Section 2;

(3) Multi-site DAQ extension with DataGrid

(4) Remote experiment on LFN technology.

These involves wide and complicated problems, so that we just started some collaborations to tackle them.

One of the most important DAQ functional extension is to cope with multiple sites and experiments, in which remote participation mechanisms and utilities would be essential. The introduction of so-called "DataGrid" technology, which will be provided by the specific middleware such as Globus toolkit or ITBL (IT-based laboratory) [12], seems to be a shorter way for revising the LABCOM data system.

These conceptual re-design for the DAQ framework would provide the universal data accessing environment among multiple sites and experiments. Simultaneously, users could obtain the proper control of data access and system security.

For advancing necessary R\&D, we just started some discussion with JAEA (JT60) and All-Japan ST research groups. To link the LHD and JT-60 DAQs with the new ITBL network middleware will be quite hopeful to bear good fruits in the near future.

The next LFN problem has already been experienced through the "Super SINET" Gigabit collaboration network even within Japan. As for ITER and its remote operation center, this solution will be the very key technology that every functions depending on it. We have another collaboration for this issue [6].

To support collaborators' communication and document sharing, the necessity of having a "collaboration groupware" tool has been pointed out. Therefore, a new Wiki page, named as "DAQ Wiki", has been opened at 


\section{http://oku.edu.mie-u.ac.jp/daq/}

to make the collaboration-related information sharable.

The output of these collaborating works would be applied and verified first in LHD experiment, then become applicable to other newer fusion projects. We aim to establish the next-generation technology to advance the outputs of this study.

\section{Acknowledgements}

The author would like to express his gratitude to Okumura H. of Mie univ. for his constant guidance about compression algorithm and informatics, and also to Ito Y. of NIFS for the dedicated works to develop the FPGA timing system. This work was partly supported by National Institute for Fusion Science under NIFS06ULHH503 and NIFS05KCHH004, and also by the Research Foundation for the Electrotechnology of Chubu (REFEC), No. E-18152.

\section{References}

[1] H. Nakanishi, M. Ohsuna, M. Kojima, S. Imazu, M. Nonomura, M. Emoto, H. Okumura, Y. Nagayama, K. Kawahata, LHD exp. group, Ultra-Wideband RealTime Data Acquisition in Steady-State Experiments, J. Plasma Fusion Res. 82 (3) (2006) 171-177.

[2] H. Nakanishi, H. Okumura, Introduction to Data Acquisition -Let's Acquire Data!-, J. Plasma Fusion Res. 81 (2) (2005) 112-119.

[3] Y. Takase, All-Japan ST Research Program, in: Abst. of 22nd JSPF Annual Meeting, Nov. 29-Dec. 2, Tokyo, Japan, 2005, pp. SV-1.

[4] K. Hiraki, M. Inaba, J. Tamatsukuri, R. Kurusu, Y. Ikuta, H. Koga, A. Zinzaki, Data Reservoir: Utilization of Multi-Gigabit Backbone Network for Data-Intensive Research, in: Proc. SC2002, 2002.

[5] M. Nakamura, M. Inaba, K. Hiraki, Fast Ethernet is sometimes faster than Gigabit Ethernet on LFN - Observation of congestion control of TCP streams, in: Proc. PDCS, Nov., 2003, pp. 854-859.

[6] T. Yamamoto, LFN: Long Fat pipe Network, http://ccpuck.nifs.ac.jp/lfn/ (2006).

[7] TOYO Corp., Network Crate Controller Model CC/NET, http://www.toyo.co.jp/daq/ ccnet/files/cat/ccnet_e.pdf (2004).

[8] ISA Corp., Remote Power Controller PDU-5115S, http://www.isa-j.co.jp/NEW/ product/pms/PDU-5115S/ (2005). 
[9] H. Nakanishi, M. Emoto, M. Kojima, M. Ohsuna, S. Komada, LABCOM group, Object-oriented data handling and oodb operation of lhd mass data acquisition system, Fusion Eng. Design 48 (1-2) (2000) 135-142.

[10] Atmark Techno, Inc., SUZAKU-S, http://suzaku-en.atmark-techno.com/series/suzaku-s (2006).

[11] M. Ohsuna, H. Nakanishi, S. Imazu, M. Kojima, M. Nonomura, M. Emoto, Y. Nagayama, H. Okumura, Unification of ultra-wideband data acquisition and realtime monitoring in LHD steady-state experiments, Fusion Eng. Des. 81 (15-17) (2006) 1753-1757.

[12] RIKEN ITBL project, ITBL Portal, http://www.itbl.jp/ (2006). 


\section{List of Figures}

1 Growth of shot-by-shot acquisition data amount in LABCOM system (top) and the numbers of diagnostics by each kind of digitizer (bottom).: At the end of 9th campaign, we had 170 shot/day, and produced about 3.0 GB/shot.

2 Former SCSI optical extenders (left) are replaced by the distant Ethernet relay and the local SCSI-USB2 conversion cable (right). We can also choose 1000BASE-SX/-LX optical links instead of UTP cables and HUBs, if the electrical isolation is carefully required.

3 Revised diagram of LABCOM DAQ and multi-tier storage system: As the 1st layer "DAQ Box" keeps raw data only on its volatile memory, they must be saved to RAID just after the acquisition is finished.

\section{List of Tables}

1 Cost comparison between SCSI optical links and local USB cable from CAMAC controller to PC: CC/NET is Ethernet I/F controller by TOYO Corp. (cost in US\$) [7]

2 Specification difference between VME/CAMAC demodulator and new FPGA one: (8)... Two of eight trigger outputs are shared for clock gating pulses. $\left(^{*}\right)$ means the total number in eleven FPGA chips.

3 Total cost comparison between previous LABCOM DAQ unit and new LABCOM/X one (cost in US\$): *() shows other digitizer cases. 

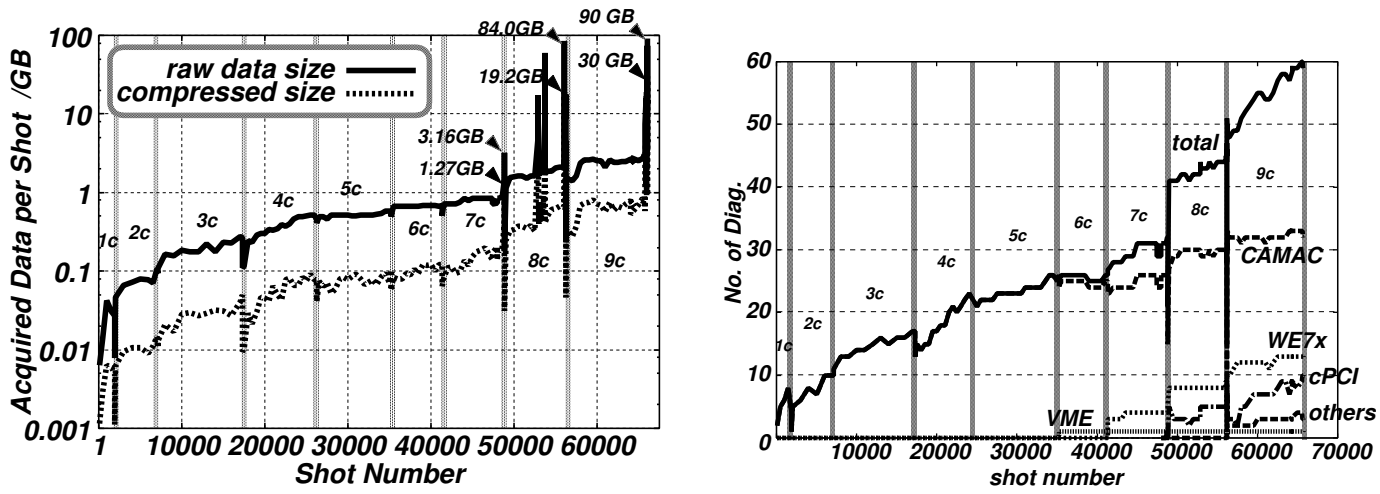

Fig. 1. Growth of shot-by-shot acquisition data amount in LABCOM system (top) and the numbers of diagnostics by each kind of digitizer (bottom).: At the end of 9th campaign, we had 170 shot/day, and produced about $3.0 \mathrm{~GB} / \mathrm{shot}$.

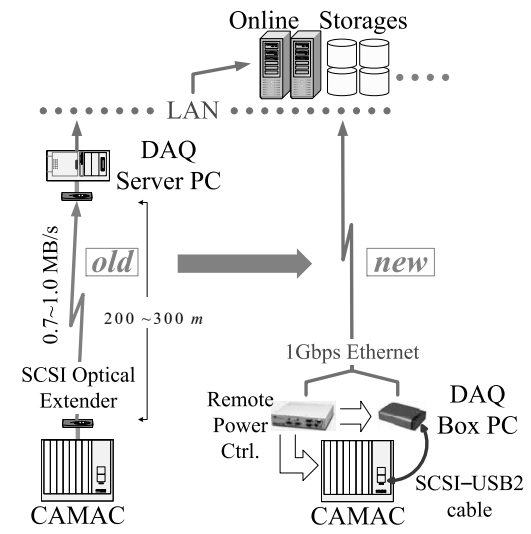

Fig. 2. Former SCSI optical extenders (left) are replaced by the distant Ethernet relay and the local SCSI-USB2 conversion cable (right). We can also choose 1000BASE-SX/-LX optical links instead of UTP cables and HUBs, if the electrical isolation is carefully required.

Table 1

Cost comparison between SCSI optical links and local USB cable from CAMAC controller to PC: CC/NET is Ethernet I/F controller by TOYO Corp. (cost in US\$) [7]

\begin{tabular}{lccc}
\hline Model & KSC3929 & Jorway73A & CC/NET \\
\hline I/F type & SCSI & SCSI-2 & Ethernet \\
prev. speed & $0.7 \mathrm{MB} / \mathrm{s}$ & $1.0 \mathrm{MB} / \mathrm{s}$ & $0.9 \mathrm{MB} / \mathrm{s}$ \\
prev. cost & $\$ 2,800$ & $\$ 2,800$ & $(\$ 4,784)$ \\
USB speed & $1.18 \mathrm{MB} / \mathrm{s}$ & $1.63 \mathrm{MB} / \mathrm{s}$ & - \\
USB cost & $\$ 56$ & $\$ 64$ & - \\
\hline
\end{tabular}


Table 2

Specification difference between VME/CAMAC demodulator and new FPGA one: (8)... Two of eight trigger outputs are shared for clock gating pulses. $\left.{ }^{*}\right)$ means the total number in eleven FPGA chips.

\begin{tabular}{lcc}
\hline spec. & VME/CAMAC & FPGA \\
\hline programmable trigger & $6(8) \mathrm{ch}$. & $6 \mathrm{ch}$. \\
divided clock & $2 \mathrm{ch}$. & $6 \mathrm{ch}$. \\
clock gating & $2 \mathrm{ch}$. & $6 \mathrm{ch}$. \\
usable gates & $150500\left(^{*}\right)$ & 1200000 \\
cost (US\$) & $\$ 6,240$ & $\$ 800$ \\
\hline
\end{tabular}

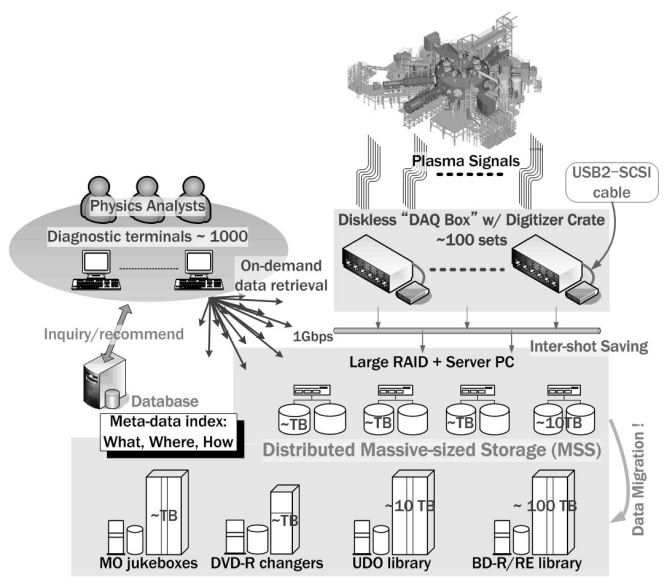

Fig. 3. Revised diagram of LABCOM DAQ and multi-tier storage system: As the 1st layer "DAQ Box" keeps raw data only on its volatile memory, they must be saved to RAID just after the acquisition is finished.

Table 3

Total cost comparison between previous LABCOM DAQ unit and new LABCOM/X one (cost in US\$): *() shows other digitizer cases.

\begin{tabular}{lcc}
\hline unit cost & $\sim$ ver.9 & LABCOM/X \\
\hline PC & $\$ 1,600$ & $\$ 800$ \\
CAMAC opti. link & $\$ 2,800$ & $\$ 64$ (USB) \\
$*$ (WE7x) & $\$ 3,040$ (fiber) & 0 (USB) \\
$*$ (PXI MXI-4/5) & $\$ 3,096$ (fiber) & $\$ 1,064$ (wire) \\
timing module & $\$ 6,240$ & $\$ 800$ \\
remote power ctrl. & - & $\$ 592$ \\
OS \& software & $\sim \$ 400$ & 0 \\
\hline Total $($ CAMAC case) & $\$ 11,040$ & $\$ 2,256$ \\
\hline
\end{tabular}

